\title{
ON A THEOREM OF APOSTOL CONCERNING MÖBIUS FUNCTIONS OF ORDER $k$
}

\author{
D. Suryanarayana
}

In 1970, Tom M. Apostol introduced a class of arithmetical functions $\mu_{k}(n)$ for all positive integral $k$, as a generalization of the Möbius function $\mu(n)=\mu_{1}(n)$ and established the following theorem: For $k \geqq 2, M_{k}(x)=\sum_{n \leqq x} \mu_{k}(n)=A_{k} x+O\left(x^{1 / k} \log x\right)$, where $A_{k}$ is a positive constant. In this paper we improve the above $O$-estimate to $O\left(x^{\left.4 k / 4 k^{2}+1\right)} \omega(x)\right)$ on the assumption of the Riemann hypothesis, where $\omega(x)=\exp \left\{A \log x(\log \log x)^{-1}\right\}, A$ being a positive absolute constant.

1. Introduction. T. M. Apostol [1] introduced the following generalization of the Möbius function $\mu(n)$. Let $k$ be a fixed positive integer. Let $\mu_{k}$, the Möbius function of order $k$ be defined by $\mu_{k}(1)=1$, $\mu_{k}(n)=0$ if $p^{k+1} \mid n$ for some prime $p, \mu_{k}(n)=(-1)^{r}$ if $n=$ $p_{1}^{k} p_{2}^{k} \cdots p_{r}^{k} \Pi_{l}>p_{l}^{a_{l}}, 0 \leqq a_{l}<k, \mu_{k}(n)=1$ otherwise. In other words, $\mu_{k}(n)$ vanishes if $n$ is divisible by the $(k+1)$ st power of some prime; otherwise, $\mu_{k}(n)$ is 1 unless the prime factorization of $n$ contains the $k$ th powers of exactly $r$ distinct primes, in which case $\mu_{k}(n)=(-1)^{r}$. When $k=1, \mu_{k}(n)$ is the usual Möbius function, $\mu_{1}(n)=\mu(n)$.

He established the following asymptotic formula (cf. [1], Theorem 1) for the summatory function $M_{k}(x)=\sum_{n \leqq x} \mu_{k}(n)$ : For $k \geqq 2$ and $x \geqq 2$

$$
\sum_{n \leqq x} \mu_{k}(n)=A_{k} x+O\left(x^{1 / k} \log x\right)
$$

where $A_{k}$ is the constant given by

$$
A_{k}=\prod_{p}\left(1-\frac{2}{p^{k}}+\frac{1}{p^{k+1}}\right)
$$

the product being extended over all primes $p$.

In this note we improve the $O$-estimate of the error term in (1) above on the assumption of the Riemann hypothesis by proving the following: For $x \geqq 3$,

$$
\sum_{n \leqq x} \mu_{k}(n)=A_{k} x+O\left(x^{4 k /\left(4 k^{2}+1\right)} \omega(x)\right)
$$

where $\omega(x)$ is given by (5) below. 
2. Lemmas. The proof of (3) is based on the following four lemmas.

Lemma 1. (cf. [5], Theorem 14-26 (A), p. 316). If the Riemann hypothesis is true, then for $x \geqq 3$,

$$
M(x)=\sum_{n \leqq x} \mu(n)=O\left(x^{1 / 2} \omega(x)\right),
$$

where

$$
\omega(x)=\exp \left\{A \log x(\log \log x)^{-1}\right\},
$$

A being an absolute positive constant.

Lemma 2 (cf. [3], Lemma 2.5). If the Riemann hypothesis is true, then for $x \geqq 3$, and $s>1$,

$$
\sum_{n \leqq x} \frac{\mu(n)}{n^{s}}=\frac{1}{\zeta(s)}+O\left(x^{\frac{1}{2}-s} \omega(x)\right),
$$

where $\zeta(s)$ is the Riemann Zeta function defined by $\zeta(s)=\sum_{n=1}^{\infty} n^{-s}$ for $s>1$.

In order to state the other two lemmas we need to recall the following terminology and notation established by E. Cohen [2]: Let $k$ be a fixed integer $\geqq 2$. A positive integer $n$ is called unitarily $k$-free, if the multiplicity of each prime divisor of $n$ is not a multiple of $k$; or equivalently, if $n$ is not divisible unitarily by the $k$ th power of any integer $>1$. By a unitary divisor of $n$, we mean as usual a divisor $d>0$ of $n$ such that $(d, n / d)=1$. The integer 1 is also considered to be unitarily $k$-free. Let $Q_{k}^{*}$ denote the set of unitarily $k$-free integers and let $q_{k}^{*}$ denote the characteristic function of the set $Q_{k}^{*}$; that is, $q_{k}^{*}(n)=1$ or 0 according as $n \in Q_{k}^{*}$ or $n \notin Q_{k}^{*}$. Let $Q_{k}^{*}(x)=\sum_{n \leqq x} q_{k}^{*}(n)$. In other words, $Q_{k}^{*}(x)$ is the number of unitarily $k$-free integers $\leqq x$. Then we have

Lemma 3 (cf. [4], Theorem 3.2). If the Riemann hypothesis is true, then for $x \geqq 3$,

$$
Q_{k}^{*}(x)=A_{k} \zeta(k) x+O\left(x^{2 /(2 k+1)} \omega(x)\right),
$$

where $\omega(x)$ is given by (5) and $A_{k}$ is given by (2). 
LEMMA 4 (cf. [1], eq. (10)). $\mu_{k}(n)=\sum_{d^{k} \delta=n} \mu(d) q_{k}^{*}(\delta)$.

3. Proof of (3). Using Lemma 4, we obtain

$$
M_{k}(x)=\sum_{n \leqq x} \mu_{k}(n)=\sum_{n \leqq x} \sum_{d^{k} \delta=n} \mu(d) q_{k}^{*}(\delta)=\sum_{d^{k} \delta \leqq x} \mu(d) q_{k}^{*}(\delta)
$$

the summation being taken over all ordered $(d, \delta)$ such that $d^{k} \delta \leqq x$.

Let $z=x^{1 / k}$. Further, let $0<\rho=\rho(x)<1$, where the function $\rho(x)$ will be suitably chosen later.

If $d^{k} \delta \leqq x$, then both $d>\rho z$ and $\delta>\rho^{-k}$ can not simultaneously hold and so from (8), we have

(9)

$$
M_{k}(x)=\sum_{\substack{d^{k} \delta \leqq x \\ d \leqq \rho z}} \mu(d) q_{k}^{*}(\delta)+\sum_{\substack{d^{k} \delta \leqq x \\ \delta \leqq \rho-k}} \mu(d) q_{k}^{*}(\delta)-\sum_{\substack{d \leqq \rho z_{k} \\ \delta \leqq \rho^{-k}}} \mu(d) q_{k}^{*}(\delta) .
$$

$$
=S_{1}+S_{2}-S_{3}, \quad \text { say }
$$

Applying Lemma 3, we obtain

$$
\begin{aligned}
S_{1} & =\sum_{d \leqq \rho z} \mu(d) \sum_{\delta \leqq x / d^{k}} q_{k}^{*}(\delta)=\sum_{d \leqq \rho z} \mu(d) Q_{k}^{*}\left(\frac{x}{d^{k}}\right) \\
& =\sum_{d \leqq \rho z} \mu(d)\left\{A_{k} \zeta(k) \frac{x}{d^{k}}+O\left(\left(\frac{x}{d^{k}}\right)^{2 /(2 k+1)} \omega\left(\frac{x}{d^{k}}\right)\right)\right\} \\
& =A_{k} \zeta(k) x \sum_{d \leqq \rho z} \frac{\mu(d)}{d^{k}}+O\left(x^{2 /(2 k+1)} \omega(x) \sum_{d \leqq \rho z} d^{-2 k /(2 k+1)}\right)
\end{aligned}
$$

since $\omega(x)$ is monotonic increasing. We have

$$
\sum_{d \leqq \rho z} d^{-2 k /(2 k+1)}=O\left((\rho z)^{1-2 k /(2 k+1)}\right)=O\left((\rho z)^{1 /(2 k+1)}\right),
$$

so that the $O$-term in $(10)$ is $O\left(\rho^{1 /(2 k+1)} z \omega(x)\right)$.

Now, applying Lemma 2 we obtain from (10),

$$
\begin{aligned}
S_{1} & =A_{k} \zeta(k) x\left\{\frac{1}{\zeta(k)}+O\left((\rho z)^{-k+1 / 2} \omega(\rho z)\right)\right\}+O\left(\rho^{1 /(2 k+1)} z \omega(x)\right) \\
& =A_{k} x+O\left(\rho^{-k+1 / 2} z^{\frac{1}{2}} \omega(x)\right)+O\left(\rho^{1 /(2 k+1)} z \omega(x)\right),
\end{aligned}
$$

since $\omega(\rho z) \leqq \omega(z)<\omega(x)$.

We have by Lemma 1 , 


$$
\begin{aligned}
S_{2} & =\sum_{\delta \leqq \rho^{-k}} q_{k}^{*}(\delta) \sum_{d \leqq(x / \delta)^{1 / k}} \mu(d)=\sum_{\delta \leqq \rho^{-k}} q_{k}^{*}(\delta) M\left(\left(\frac{x}{\delta}\right)^{1 / k}\right) \\
& =O\left(\sum_{\delta \leqq \rho^{-k}} q_{k}^{*}(\delta)\left(\frac{x}{\delta}\right)^{1 / 2 k} \omega\left(\left(\frac{x}{\delta}\right)^{1 / k}\right)\right) \\
& =O\left(x^{1 / 2 k} \omega(x) \sum_{\delta \leqq \rho-k} q_{k}^{*}(\delta) \delta^{-1 / 2 k}\right)
\end{aligned}
$$

Now, by Lemma 3 and partial summation, we obtain

$$
\sum_{\delta \leqq \rho^{-k}} q_{k}^{*}(\delta) \delta^{-1 / 2 k}=O\left(\left(\rho^{-k}\right)^{1-1 / 2 k}\right)=O\left(\rho^{-k+1 / 2}\right)
$$

Hence by (12), we have

$$
S_{2}=O\left(\rho^{-k+1 / 2} z^{1 / 2} \omega(x)\right)
$$

Also, by Lemmas 1 and 3, we obtain

$$
\begin{aligned}
S_{3} & =\left(\sum_{d \leqq \rho z} \mu(d)\right)\left(\sum_{\delta \leqq \rho} q_{k}^{*}(\delta)\right)=O\left(\rho^{1 / 2} z^{1 / 2} \omega(\rho z) \rho^{-k}\right) \\
& =O\left(\rho^{-k+1 / 2} z^{1 / 2} \omega(x)\right) .
\end{aligned}
$$

Hence by (9), (11), (13) and (14), we obtain

$$
M_{k}(x)=A_{k} x+O\left(\rho^{-k+1 / 2} z^{1 / 2} \omega(x)\right)+O\left(\rho^{1 /(2 k+1)} z \omega(x)\right)
$$

Now, choosing $\rho=z^{-(2 k+1) /\left(4 k^{2}+1\right)}$, we see that $0<\rho<1$ and

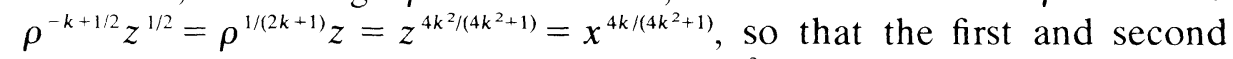
$O$-terms in (15) are both equal to $O\left(x^{4 k /\left(4 k^{2}+1\right)} \omega(x)\right)$. Hence (3) follows from (15).

In conclusion we would like to make the following two remarks:

REMARK 1. The $O$-estimate in (3) is uniform in $x$ and $k$.

REMARK 2. Since we have obtained improvement in Apostol's Theorem (1) on the assumption of the Riemann hypothesis by making use of Lemma 3 or Theorem 3.2 of [4], it might appear that it is possible to obtain improvement in (1) even without any hypothesis, by making use of Theorem 3.1 of [4]. However, this does not seem possible, at least by our method. 


\section{REFERENCES}

1. Tom M. Apostol, Möbius functions of order k, Pacific J. Math., 32 (1970), 21-27.

2. E. Cohen, Some sets of integers related to the $k$-free integers, Acta Sci. Math., (Szeged) 22 (1961), 223-233.

3. D. Suryanarayana and V. Siva Rama Prasad, The number of $k$-free divisors of an integer, Acta Arith., 17 (1971), 345-354.

4. D. Suryanarayana and R. Sita Rama Chandra Rao, Distribution of unitarily $k$-free integers, J. Austral. Math. Soc., 20 (Series A) (1975), 129-141.

5. E. C. Titchmarsh, The Theory of the Riemann Zeta function, Clarendon Press, Oxford, 1951.

Received November 2, 1976.

MEMPhis State University

MEMPHIS, TN 38152

AND

ANDHRA UNIVERSITY 
